\title{
A Comprehensive Transcriptomics Analysis Reveals Long Non-Coding RNA to Be Involved in the Key Metabolic Pathway in Response to Waterlogging Stress in Maize
}

\author{
Feng Yu ${ }^{1,2,+} \mathbb{D}$, Zengdong Tan ${ }^{2,+}$, Tian Fang ${ }^{2}$, Kaiyuan Tang ${ }^{2}$, Kun Liang ${ }^{2}$ and Fazhan Qiu ${ }^{2, *}$ \\ 1 State Key Laboratory of Biocatalysis and Enzyme Engineering, School of Life Sciences, Hubei University, \\ Wuhan 430062, China; yufeng@hubu.edu.cn \\ 2 National Key Laboratory of Crop Genetic Improvement, Huazhong Agricultural University, Wuhan 430070, \\ China; zdtan1217@gmail.com (Z.T.); ft13026029102@163.com (T.F.); TKYcircle@163.com (K.T.); \\ kl353739@126.com (K.L.) \\ * Correspondence: qiufazhan@mail.hzau.edu.cn; Tel.: +86-027-872-86870; Fax: +86-027-872-80016 \\ + The authors had equal contributions.
}

Received: 29 January 2020; Accepted: 27 February 2020; Published: 29 February 2020

check for updates

\begin{abstract}
Waterlogging stress (WS) in a dynamic environment seriously limits plant growth, development, and yield. The regulatory mechanism underlying WS conditions at an early stage in maize seedlings is largely unknown. In the present study, the primary root tips of B73 seedlings were sampled before ( $0 \mathrm{~h}$ ) and after $(2 \mathrm{~h}, 4 \mathrm{~h}, 6 \mathrm{~h}, 8 \mathrm{~h}, 10 \mathrm{~h}$, and $12 \mathrm{~h}) \mathrm{WS}$ and then subjected to transcriptome sequencing, resulting in the identification of differentially expressed protein-coding genes (DEpcGs) and long non-coding RNAs (DElncRs) in response to WS. These DEpcGs were classified into nine clusters, which were significantly enriched in several metabolic pathways, such as glycolysis and methionine metabolism. Several transcription factor families, including AP2-EREBP, bZIP, NAC, bHLH, and MYB, were also significantly enriched. In total, 6099 lncRNAs were identified, of which 3190 were DElncRs. A co-expression analysis revealed lncRNAs to be involved in 11 transcription modules, 10 of which were significantly associated with WS. The DEpcGs in the four modules were enriched in the hypoxia response pathways, including phenylpropanoid biosynthesis, MAPK signaling, and carotenoid biosynthesis, in which 137 DElncRs were also co-expressed. Most of the co-expressed DElncRs were co-localized with previously identified quantitative trait loci associated with waterlogging tolerance. A quantitative reverse transcription-polymerase chain reaction analysis of DEpcG and DElncR expression among the 32 maize genotypes after $4 \mathrm{~h}$ of WS verified significant expression correlations between them as well as significant correlation with the phenotype of waterlogging tolerance. Moreover, the high proportion of hypoxia response elements in the promoter region increased the reliability of the DElncRs identified in this study. These results provide a comprehensive transcriptome in response to WS at an early stage of maize seedlings and expand our understanding of the regulatory network involved in hypoxia in plants.
\end{abstract}

Keywords: maize; waterlogging stress; mRNA; long non-coding RNA; co-expression network

\section{Introduction}

Waterlogging is one of major abiotic stresses that limits the productivity of terrestrial plants and has increasingly and frequently occurred in recent years due to global climate change [1,2]. Oxygen deprivation is the key feature of waterlogging stress, as plants alter their metabolism, physiology, and development to acclimatize to the low levels of oxygen [3]. The cause of these changes is the 
dramatic reprogramming of gene expression and RNA molecules, including coding RNA (mRNA) and non-coding RNA (ncRNA) that has been quantified with the development of deep high-throughput sequencing [4,5]. Numerous investigations into Arabidopsis [6-8], rice [9], maize [10,11], poplar (Populus $\times$ canescens) [12], and cotton (Gossypium hirsutum) [13] have been conducted to elucidate changes in the gene transcription of mRNA in response to low oxygen or flooding stress; these studies have revealed the similarity in transcriptome responses among species [14,15]. However, knowledge of ncRNA involved in hypoxia responses is largely unknown.

Long non-coding RNA (lncRNA) is defined as a transcript that is longer than 200 nucleotides (nt) in length and lacks coding potential. These RNAs have also been reported to affect the expression of other genes [16]. Thousands of lncRNAs have been identified in many plant species, such as Arabidopsis [17,18], rice (Oryza sativa) [19,20], maize (Zea mays L.) [21], cotton (Gossypium spp.) [5], and castor bean (Ricinus communis) [22], with the rapid development of deep RNA-seq techniques. Recent studies have demonstrated that lncRNAs participate in diversified biological functions in different organisms $[23,24]$. Nevertheless, only a few lncRNAs have been well characterized in plants, including COOLAIR and COLDAIR in the regulation of flowering in Arabidopsis [25,26], INDUCED BY PHOSPHATE STARVATION1, which is involved in phosphate uptake [27,28]; Enod40, which is involved in nodulation [29,30]; LDMAR, which is required for pollen development in rice [31]; LAIR, which increases rice grain yield [32]; and $E f-c d$, which shortens rice maturity duration without a yield penalty [33]. Remarkably, accumulated evidence has demonstrated the involvement of lncRNAs in biotic and abiotic stress responses in plants [34]. For example, the pathogen-responsive lncRNA ALEX1, which is highly induced by Xanthomonas oryzae pv. Oryzae infection in rice, has been shown to be involved in the jasmonic acid pathway and to offer resistance to bacterial blight [35]; a nucleus-localized lncRNA DRIR was found to be a positive regulator enhancing plant responses to drought and salt stress in Arabidopsis [36]; a total of 144 lncRNAs that respond to cadmium stress in rice at an early stage were identified to regulate the genes of cysteine-rice peptide metabolism in cis [37]; and nitrogen deficiency in Populus triggers 126 lncRNAs to change their expression and become involved in a complex regulatory network [38]. These findings collectively suggest the diversified functions of lncRNAs, as well as their importance in plant development and stress responses.

Hypoxia is a common phenomenon that occurs in plant tissues, especially in waterlogged organs. Studies on mammals have demonstrated that lncRNAs are key regulators involved in hypoxic stress, such as lncRNA $H 19$ protecting $\mathrm{H} 9 \mathrm{c} 2$ cells against hypoxia-induced injury by targeting MicroRNA-139 [39] and contributing to hypoxia-induced CPC injury by suppressing Sirt1 [40], hypoxia-induced HOTAIR expression regulated by HIF1 $\alpha$ (hypoxia-inducible factor) contributing to its roles in tumorigenesis [41], TUG1 playing an important role in hypoxia-induced myocardial cell injury by regulating the miR-145-5p-Binp3 axis [42], and hypoxia-induced lncRNA NUTF2P3-001 contributing to the tumorigenesis of pancreatic cancer by derepressing the miR-3923/KRAS pathway [43]. Little knowledge of lncRNA's involvement in hypoxic stress in plant species has been acquired, with the exception of the observation that root-specific $A t R 8$ increases accumulation under hypoxic conditions [44]. Thus, the global identification and characterization of lncRNAs involved in hypoxia regulation in plants are needed.

Maize is an upland crop and sensitive to waterlogging stress (WS). To date, only three studies have focused on identifying the important regulators and processes involved in WS in maize through transcriptome sequencing (RNA-seq) analysis $[10,11,45]$. These studies focused on their central role in the adaptation process after long-term stress, but they did not provide a global view of the transcriptome at an early response stage. Remarkably, no experiment was performed to investigate the lncRNA response to WS in maize. In the present study, maize inbred line B73 was subjected to WS conditions at the second leaf stage, and the root tips of B73 were sampled before treatment $(0 \mathrm{~h}$, WS_0h) and after $2 \mathrm{~h}$ (WS_2h), $4 \mathrm{~h}$ (WS_4h), $6 \mathrm{~h}$ (WS_6h), $8 \mathrm{~h}$ (WS_8h), $10 \mathrm{~h}$ (WS_10h), and $12 \mathrm{~h}$ (WS_12h) to investigate the global expression of the protein coding gene (PCgenes) and lncRNAs at an early stage. Expression profiling and co-expression network analyses of PCgenes and lncRNAs were conducted to 
identify the key metabolic pathway and lncRNAs involved in WS responses. These results expand our understanding of the transcriptome response of PCgenes and the potential functions of lncRNAs under waterlogged root tips; these results also provide a valuable resource for elucidating the molecular regulatory network responding to WS conditions.

\section{Materials and Methods}

\subsection{Plant Materials and Growth Conditions}

Seeds of maize inbred line B73 were planted in a greenhouse with a controlled temperature $\left(\sim 28^{\circ} \mathrm{C} / 22{ }^{\circ} \mathrm{C}\right.$ day/light cycle), a $14 \mathrm{~h} / 10 \mathrm{~h}$ light/dark cycle (light time: 7:00 am-9:00 pm), and average humidity (60\% average). The growth substrate and waterlogging treatments were conducted as previously described in [46]. Briefly, ten uniform seedlings were planted in a plastic pot, and treatments were applied by maintaining a $2-3 \mathrm{~cm}$ water layer above the substrate at the second leaf stage, and stress was treated at 8:00 am. To prepare the sample for sequencing, ten root tips $(\sim 2 \mathrm{~cm})$ of the primary roots in each plot were collected before treatment $(0 \mathrm{~h}$, control) and after $2 \mathrm{~h}, 4 \mathrm{~h}, 6 \mathrm{~h}, 8 \mathrm{~h}, 10 \mathrm{~h}$, and $12 \mathrm{~h}$ stress and mixed as one replication. Three replications were prepared at each time point. In total, 21 samples were snap frozen in liquid nitrogen and stored at $-80^{\circ} \mathrm{C}$ for total RNA isolation.

\subsection{RNA Extraction, Library Construction, and Sequencing}

Total RNA was extracted using TRIZOL reagent (Invitrogen, Gaithersburg, MD, USA) and purified with an RNeasy mini kit (QIAGEN, Germantown, MD, USA) according to the manufacturer's instructions. RNA integrity was detected using gel electrophoresis. High-quality RNA was used for library construction using the Illumina TruSeq Stranded RNA Kit (Illumina, San Diego, CA, USA) following the manufacturer's recommendations. Transcriptome sequencing of the prepared libraries was performed on an Illumina HisSeq 4000 system with paired-end 150-bp reads (Shanghai Personal Biotechnology, China).

\subsection{Quantification and Standardization of the PCgenes and IncRNAs in the Transcriptome}

A total of 21 RNA-Seq data sets were generated from this study. All raw reads that had been deposited into the SRA (accession number: SRP249592) and GEO (accession number: GSE146136) were assessed for quality using the program of FastQC (V0.11.3) and filtered using Trimmomatic (V0.38) [47] to obtain clean data. The clean reads of all samples were aligned to the maize B73 reference genome (Ref_Gen4) downloaded from MaizeGDB using Hisat2 (v2.0.5) [48] with the default parameters. The featureCount (V1.6.4) [49] in the Rsubread package was used for quantification and standardization to obtain the read count and TPM (transcripts per kilobase per million) value of each expressed gene, in which the TPMs represented the expression level of each transcript.

For identification of lncRNAs, the "bowtie2" (V2.3.4.1) [50] was applied to build index files. The assembly of each transcriptome datum was independently conducted using CUFFLINKS (V2.2.0) [51]; a merged.gtf file was obtained from merging these data together. "RSEM" (V1.3.0) [52] was utilized to normalize and calculate the TPM value. To identify high confidence lncRNAs, the Perl script LncRNA_Finder.pl was used to filter the lengths of the isoforms, the number of orfs, and Coding Potential Calculator 2 (CPC2) to eliminate potential protein-coding RNAs [21]. According to the annotation information of the merged.gtf file assembled by CUFFLINKS, the isoforms marked with $\mathrm{u}$ and $\mathrm{x}$ were filtered, and finally, the isoforms with an expression amount of 0 or a mean TPM $<0.5$ under the same conditions were removed.

\subsection{Differential Expression and Pathway Analysis}

A differential expression analysis between pairs of samples was performed using the DESeq2 $\mathrm{R}$ package [53] to identify differentially expressed PCgenes (DEpcGs) and differentially expressed lncRNAs (DElncRs) for transcriptomes containing biological replicates, and the adjusted $p$-values were calculated 
using the Benjamini and Hochberg method [54] to control the false discovery rate. The standard for screening DEpcGs and DElncRs was set as 1) an adjusted $p<0.05$ and 2) $\log _{2}$ (a foldchange) $>1$, or $\log _{2}$ (a foldchange) $<-1$. The different expression profiles between the stress conditions (WS_2h, WS_4h, WS_6h, WS_8h, WS_10h, and WS_12h) and the normal condition (WS_0h) were analyzed. The mfuzz package [55] was used to divide the different time expression patterns into differential clusters. An enrichment analysis of DEpcGs was conducted using KEGG enrichment analysis. The online software AgriGO V2.O (http://systemsbiology.cau.edu.cn/agriGOv2/index.php) [56] was applied to analyze the gene ontology (GO). Heat maps and Venn diagrams were drawn using R packages.

\subsection{Expression Network Construction}

To investigate the co-expression profiling between PCgenes and lncRNA, all transcripts of highly-confident lncRNAs and DEpcGs were subjected to the weighted gene co-expression network analysis (WGCNA) method [57] to construct an expression matrix, and genes with similar expression patterns were clustered into the same module. The relationships between the transcripts in the module and the samples were investigated, and the important modules that were significantly associated with the sample traits (WS_0h, WS_2h, WS_4h, WS_6h, WS_8h, WS_10h, and WS_12h) were identified. The genes in the modules were then subjected to KEGG pathway enrichment. Finally, visualization of the co-expression network was performed using the Cytoscape (v3.5.0) [58].

\subsection{Quantitative Real-Time (RT) PCR}

The extracted total RNA of all samples subjected to transcriptome analysis was also used for quantitative real-time PCR. The total RNA was purified using RNase-free DNase (Invitrogen, Gaithersburg, MD, USA), and single-stranded cDNA was synthesized using recombinant M-MLV reverse transcriptase (Invitrogen) according to the manufacturer's protocol. qRT-PCR was conducted using gene-specific primers (Table S7) in a $25 \mu \mathrm{L}$ reaction with a $2 \times \mathrm{iTaq}^{\mathrm{TM}}$ Universal SYBR Green Supermix (BioRad, Hercules, CA, USA) under the following conditions: initial denaturation at $95^{\circ} \mathrm{C}$ for $5 \mathrm{~min}$, followed by 40 cycles at $95^{\circ} \mathrm{C}$ for $15 \mathrm{~s}, 58{ }^{\circ} \mathrm{C}$ for $10 \mathrm{~s}$, and $72{ }^{\circ} \mathrm{C}$ for $20 \mathrm{~s}$. The internal reference ZmActin1 was utilized to normalize the expression data. Relative expression levels were calculated according to the $2^{-\Delta \Delta C T}$ (cycle threshold) method [59].

\subsection{Verification of the Co-Expression Modules of DEpcGs and DElncRs in Different Inbred Lines}

Thirty-two maize inbred lines in the association panel [48] were randomly selected and subjected to waterlogging stress during the second leaf stage, as mentioned above. The root samples of each line were collected before $(0 \mathrm{~h})$ and after $(4 \mathrm{~h})$ stress, and total RNA was extracted for further use. An expression analysis of DEpcGs and DElncRs was conducted using the qRT-PCR technique. The expression correlation between DEpcGs and DElncRs and significant $p$-values were calculated using the R software (R Development Core Team 2013; version 3.6.3; http://www.r-project.org/).

\subsection{Conserved Motif Discovery}

To discover the potential conserved motif in the promoter region of DElncRs, the DElncRs in each comparison were screened again based on stricter criteria: 1 ) $\log _{2}$ (a foldchange) $>1.5 ; 2$ ) a baseMean of each comparison $>50$; 3) $p<0.05$. The 1500 bp upstream sequences in front of the transcript start sites of the screened DElncRs were downloaded from the B73 reference genome, and the resulting sequences were submitted to the PlantCARE database (accessed on: November 27, 2019) [60] to search for the conserved motif involved in stress and hormone response. 


\section{Results}

\subsection{The Time-Course Transcriptomic Profiles of Seedling Root Tips Exposed to WS Conditions}

To investigate the gene expression dynamics of the root tips of the B73 seedling in response to WS, all the samples (WS_0h, WS_2h,WS_4h,WS_6h,WS_8h,WS_10h, and WS_12h) were subjected to transcriptome sequencing (RNA-seq). After high-depth RNA-seq, about one hundred million pair-end reads from each sample were generated, and the average rate of uniquely mapped reads aligned to maize reference genome [61] for all samples was 85.6\% (Table S1). Uniquely mapped reads were used to calculate the normalized transcription level as transcripts per kilobase per million (TPM), and the average TPM value $>1$ of all the 21 samples of these genes were considered as an expression. In total, 23,175 expressed genes were detected in at least one sample (Table S2).

For an investigation of the transcriptome differences of the PCgenes between normal (WS_0h) and stressed (WS_2h,WS_4h,WS_6h,WS_8h,WS_10h, and WS_12h) conditions, the differentially expressed PCgenes (DEpcGs) were analyzed (Supplemental Data Set S1). The up-regulated DEpcGs ranged from 1234 at WS-2h to 2732 at WS-6h and had similar gene numbers at WS-2h/WS-4h and WS-6h/WS-10h/WS-12h, respectively (Figure 1A). A total of 330 DEpcGs, including genes coding for enzymes involved in glycolysis and fermentation (Zm00001d028759 encoded Pyruvate decarboxylase; Zm00001d037689 encoded hexokinase7) and ethylene signaling related genes (Zm00001d027622 encoded the C3HC4-type RING finger protein), were detected at all time points, and time point-specific differentially expressed genes (DEGs) numbered more than 70 in each sample. The down-regulated DEpcGs ranged from 555 at WS-2h to 2375 at WS-6h, which demonstrated the rapid growth of DEGs at WS-4h and a reduction at WS-8h (Figure 1B). These results suggest that the DEGs gradually accumulated in response to WS conditions in the early stage of stress ( 2 h, 1789 DEGs; 4 h, 3312 DEGs; $6 \mathrm{~h}, 5107$ DEGs) and stably expressed during the late stage (10 h, 4654 DEGs; 12 h, 4506 DEGs).

To globally provide an expression profile after WS conditions, expression models based on a $\log _{2}$ (foldchange) of DEpcGs were created and were neatly divided into nine clusters (Cluster1-Cluster9), including 4261 genes ranging from 351 to 665 in each cluster (Figure 1C,D). The KEGG enrichment analysis demonstrated that three clusters, cluster1, cluster3, and cluster6, were significantly (FDR < 0.05) enriched in a specific pathway (Figure 1D). Four genes in Cluster1 (Zm00001d048702 encoded indolin-2-one monooxygenase-like protein, Zm00001d048703 encoded Cytochrome P450 71C1, Zm00001d048705 encoded cytochrome P450 71C3, and Zm00001d048710 encoded benzoxazinone synthesis 2) were enriched in benzoxazinoid biosynthesis, and 11 genes in Cluster2, including Zm00001d052494 encoded pyruvate kinase and Zm00001d034256 encoded phosphohexose isomerase 1 , were enriched in glycolysis/gluconeogeneous (Figure S1). Cluster6 had the largest amount of DEpcGs and was enriched in four different pathways, including 24 genes (such as Zm00001d022282, Zm00001d022283, and Zm00001d024752 encoded peoxidase) involved in phenylpropanoid biosynthesis, 9 genes (such as Zm00001d017275, Zm00001d017276, and Zm00001d017279 encoded phenylalanine ammonia-lyase) involved in phenylalanine metabolism, 12 genes (such as Zm00001d024850, Zm00001d024851, and Zm00001d024852 encoded 1-aminocyclopropane-1-carboxylate oxidase) involved in cysteine and methionine metabolism, and 10 genes involved in ( $\alpha$-) Linolenic acid metabolism (Figure S1). These significantly enriched metabolism pathways were tightly linked to the WS response. Moreover, a Gene Ontology (GO) analysis of Cluster1 to Cluster 9 also showed GO terms of the biological processes associated with WS conditions being significantly enriched in these clusters, except for Cluster9 (Figures S2-S9). For example, the cell wall related processes in Cluster1 and Cluster2 (Figures S2 and S3), the energy metabolism-related process in Cluster3 (Figure S4), the regulation related process in Cluster4 (Figure S5), and the stress response-associated process in Cluster6 (Figure S7) were significantly enriched. 


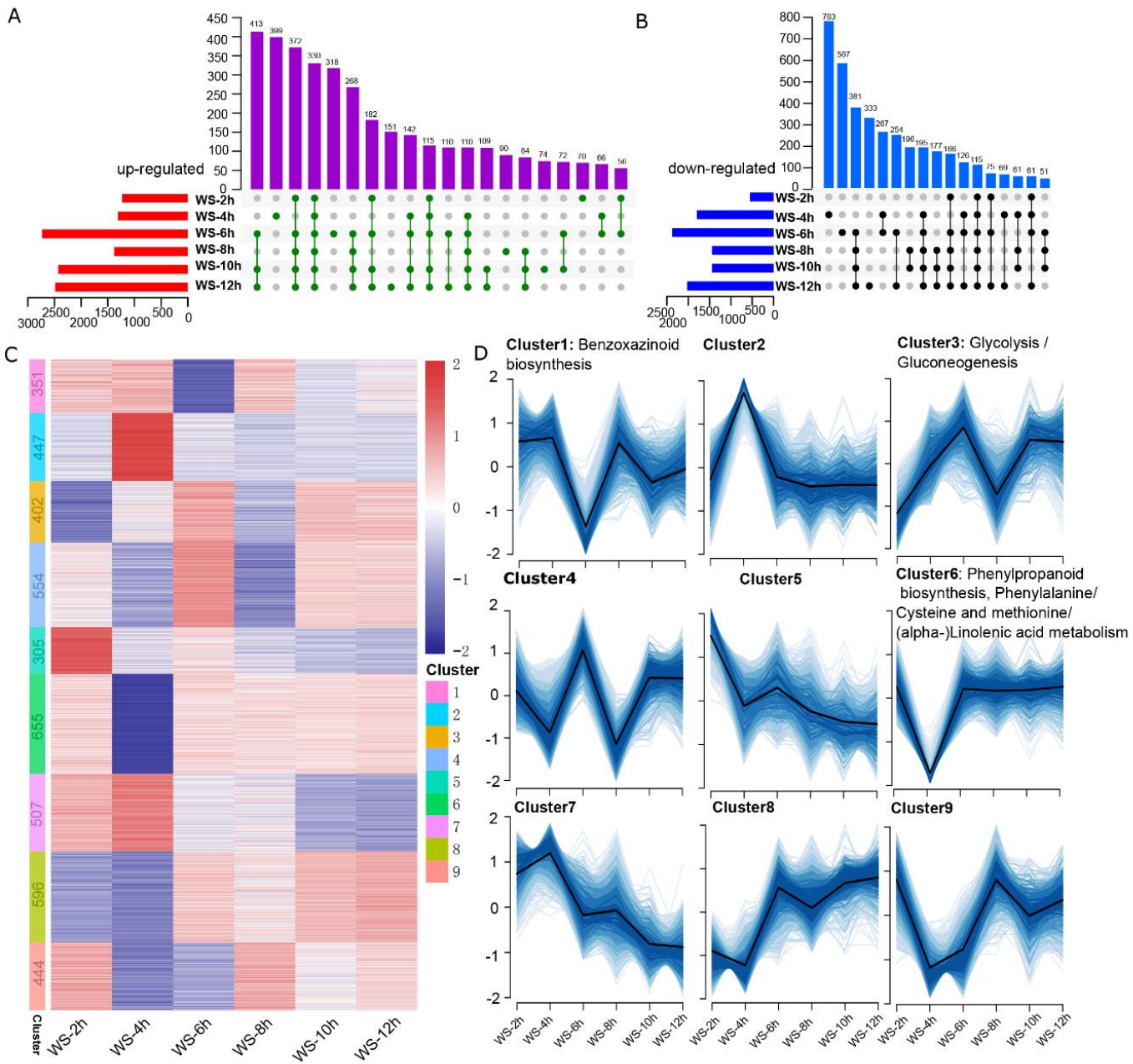

Figure 1. The characteristics of differentially expressed protein coding genes (DEpcGs) after waterlogging stress (WS). The Venn diagram shows the number of up-regulated DEpcGs (A) and down-regulated DEpcGs (B) after $2 \mathrm{~h}$ (WS_2h), 4 h (WS_4h), $6 \mathrm{~h}$ (WS_6h), 8 h (WS_8h), 10 h (WS_10h), and $12 \mathrm{~h}$ (WS_12h) of stress. These DEpcGs are divided into 9 clusters based on the 'mfuzz' package in the R software and were displayed using a heatmap $(\mathbf{C})$ and their expression tendency $(\mathbf{D})$. The numbers in the heatmap represent the DEpcG amounts in each cluster, and the significantly enriched KEGG pathways in Cluster1, Cluster3, and Cluster6 are shown.

\subsection{Differential Response of the Transcription Factor Families Involved in WS Conditions}

Transcription factors play vital roles in regulating gene expression under normal and stress conditions. To investigate the characterization of the transcription factors (TF) involved in WS conditions, an enrichment analysis of all the TF families of maize downloaded from PlantTFDB was conducted, which revealed that $15 \mathrm{TF}$ families were significantly enriched during at least one time point (Figure 2A). The enrichment of NAC, including four up-regulated genes, Zm00001d000112, Zm00001d012527, Zm00001d013003, and Zm00001d019027; MYB, including seven down-regulated genes such as Zm00001d011669 and Zm00001d035918; and AP2-EREBP, such as Zm00001d031728 and Zm00001d040651, were observed at six time points, and bHLH, bZIP, AUX-IAA, and SRS were enriched at at least three time points. A total of 45 NACs were differentially expressed after WS, about half of which were up-regulated (Figure 2B). Of the $48 \mathrm{bHLHs}, 24$ genes including five genes (Zm00001d013130, Zm00001d027987, Zm00001d042263, Zm00001d043699, and Zm00001d04512) at six time points were also up-regulated (Figure 2C). The up-regulated AP2-EREBP (Figure 2D) and bZIP including five up-regulated genes, Zm00001d005962, Zm00001d0012296, Zm00001d017911, Zm00001d039065, and Zm00001d041920 (Figure 2F), were significantly enriched, whereas the down-regulated MYBs were also 
significantly enriched (Figure 2E). Investigations in plants have shown that group VII AP2-EREBP genes control flooding response and low-oxygen tolerance [62]. Of the 19 group VII AP2-EREBP genes in maize [46], eight genes were significantly up-regulated, implying that their potential functions regulate hypoxia response. These data demonstrated the discrepant performance of TFs under WS conditions.

A

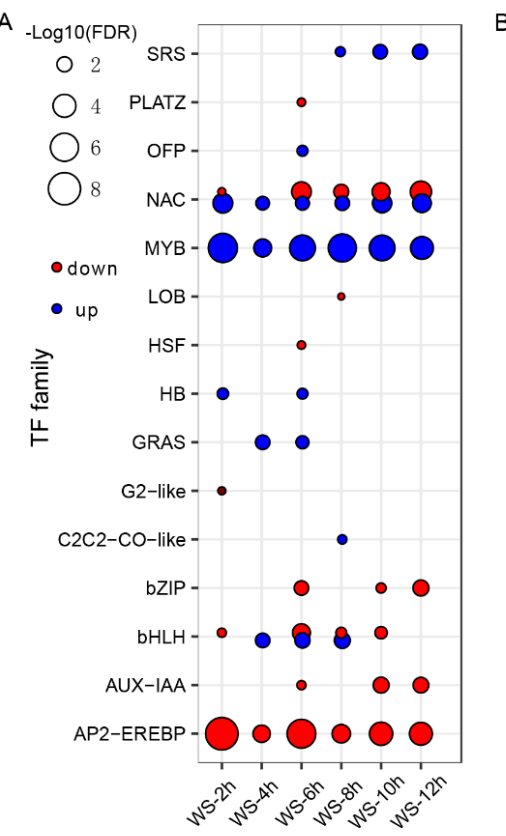

D

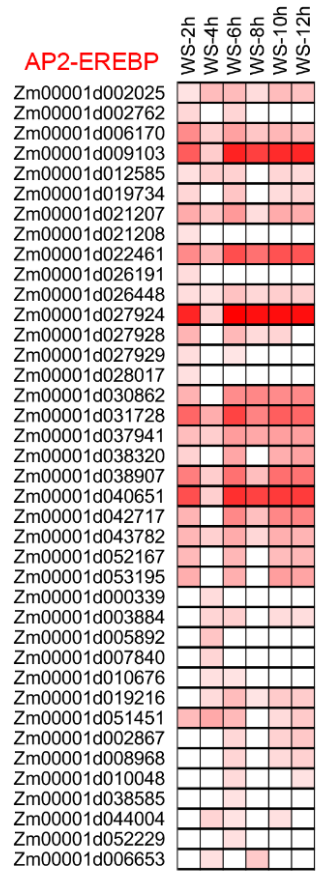

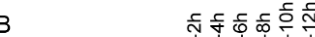

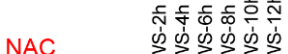

Zmo0001d003052 Zmo00010003414

Zm00001d006053

$\mathrm{Zm00001d016814}$

Zm00001d025861

Zm00001d035084

Zm00001d036050

Zm000001d043921

Zm00001d045463

Zm00001d046126

m00001049678

Zm00001d051956

$Z m 00001 d 000112$

m00001d002285

Zm00001d005208

Zm00001d012527

Im0001d013003

Zm00001d01920

Im00001d023294

Zm00001d023669

Zm00001d028999

Zm00001d040318

Im00001d042609

Zm00001d042609

$\mathrm{m} 00001039506$

Zm00001d010144

Zm00001d024543

Zm00001d0483

Zm00001d051140

m0001024268

Zm00001d034277

Zm00001d010227

$Z m 00001 d 014405$

Zm00001d036364

Zm00001d022517

Zm00001d02899

Zm00001d04986

E

MYB

Zm00001d006585 Zm00001d008528 Zm00001d011669 Zm00001d019712 Zm00011027623 m00001d030678 Zm00001d031270 Zm00001d033815 Zm00001d035918 Zm00001d04157 Zm00001d048383 Zm0000104052804 Zm00001d0024 Zm00001d01090 Zm00001d0212 Zm00001d02619 Zm00001d03893 Zm00001d040527 Zmo0001d041853 Zm00001d045560 Zm00001d009435 Zm00001d017243 Zm00001d02225 Zm00001d02393 $Z m 00001 d 024784$ Zm00001d03269 Zmo0001d05114 Zm00001d02620 Zm00001d04062 Zm00001d042665 Zm00001d021537

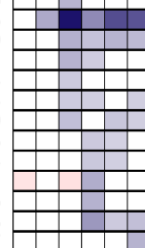

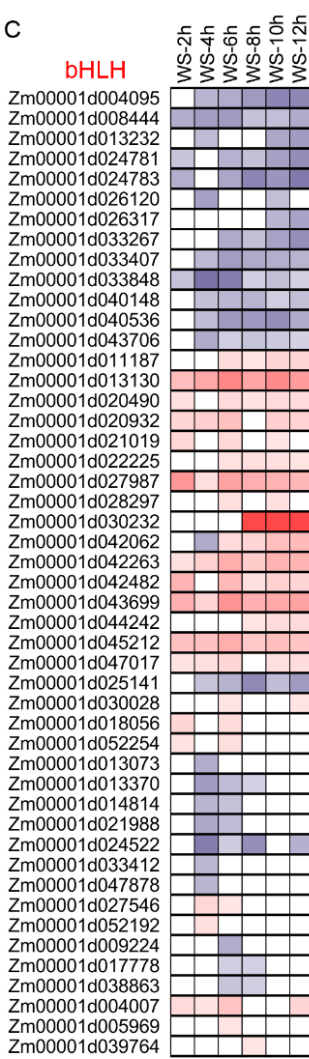

Zm00001d005969

$\mathrm{F}$
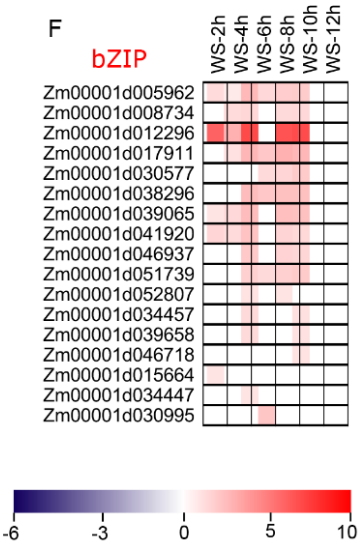

Figure 2. The enrichment analysis of transcription factors (TFs) after waterlogging stress (WS). The TF members of each family were downloaded from PlantTFDB (http://planttfdb.cbi.pku.edu.cn/). (A) The bubble chart shows the significantly enriched TF family in up- and down-regulated catalogs. (B) The gene list of NAC TFs enriched after WS treatment. (C) The gene list of bHLH TFs enriched after WS treatment. (D) The gene list of AP2-EREBP TFs enriched after WS treatment. (E) The gene list of MYB TFs enriched after WS treatment. (F) The gene list of bZIP TFs enriched after WS treatment. 


\subsection{Identification and Characterization of IncRNAs Responding to WS in the Root Tips of Maize Seedling}

A total of 6098 transcripts (Table S3) were finally identified as lncRNAs in B73 seeding root tips, including 5662 lincRNAs and 436 lncNATs (Table S4). To clarify the characteristics of lncRNAs in maize root tips, the lengths, exon numbers, and expression levels of lincRNAs, lncNATs, and PCgenes were compared. The average lengths of the lincRNAs and lncNATs were $1044 \mathrm{nt}$ and $1435 \mathrm{nt}$, respectively, which were shorter than PCgenes (3807 nt) (Figure 3A). Approximately $91 \%$ of the lincRNAs and $92 \%$ of lncNATs contained less than two exons, while only $42 \%$ of PCgenes had one or two exons (Figure 3B). Remarkably, about $29 \%$ of PCgenes had more than ten exons, whereas few lincRNAs and lncNATs were detected with this characteristic. Moreover, the normalized TPM demonstrated that the average TPM of PCgenes was higher than that of lincRNAs and lncRNAs (including lincRNAs and lncNATs) but lower than that of lncNATs (Figure 3C).
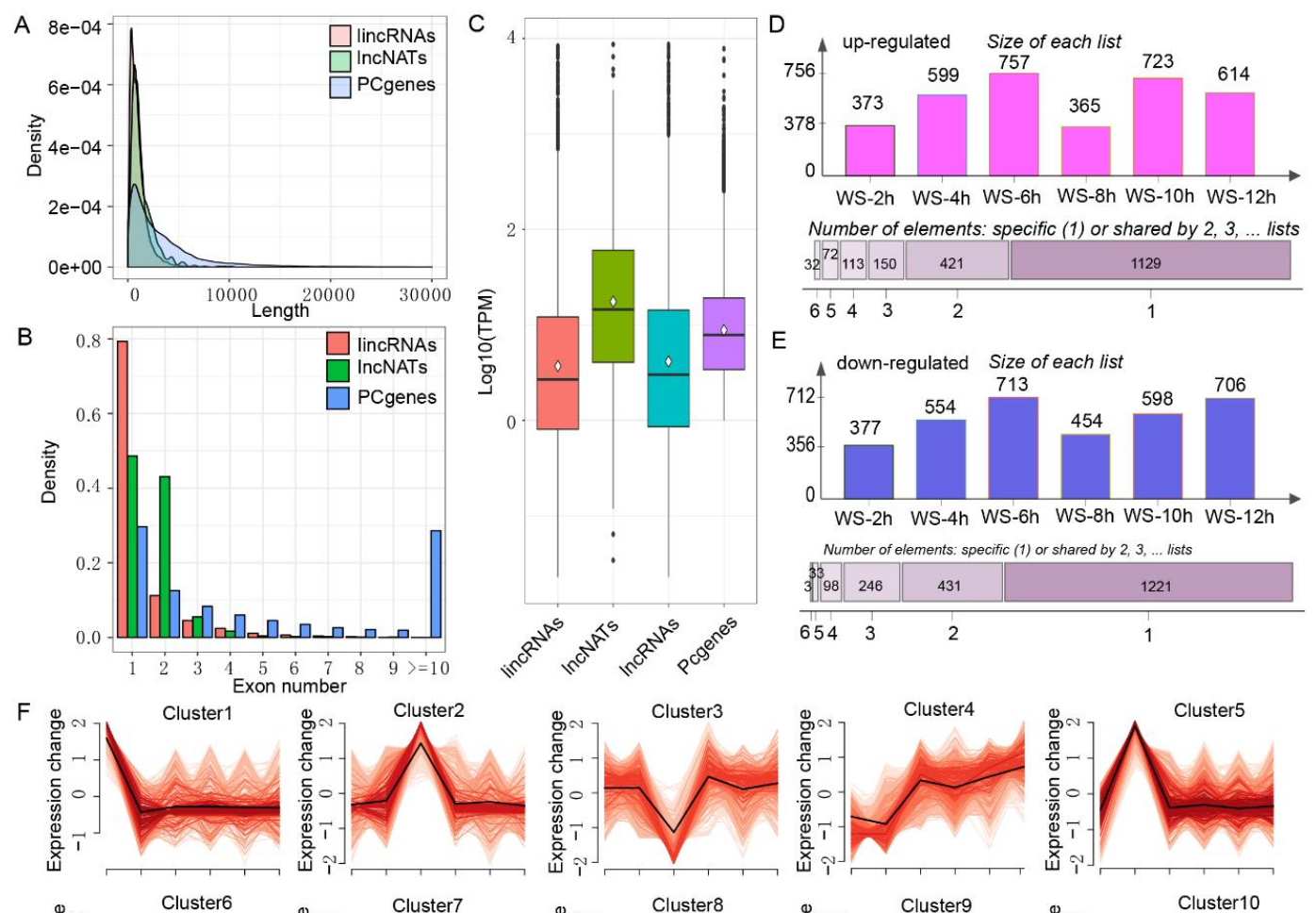

down-regulated Size of each list
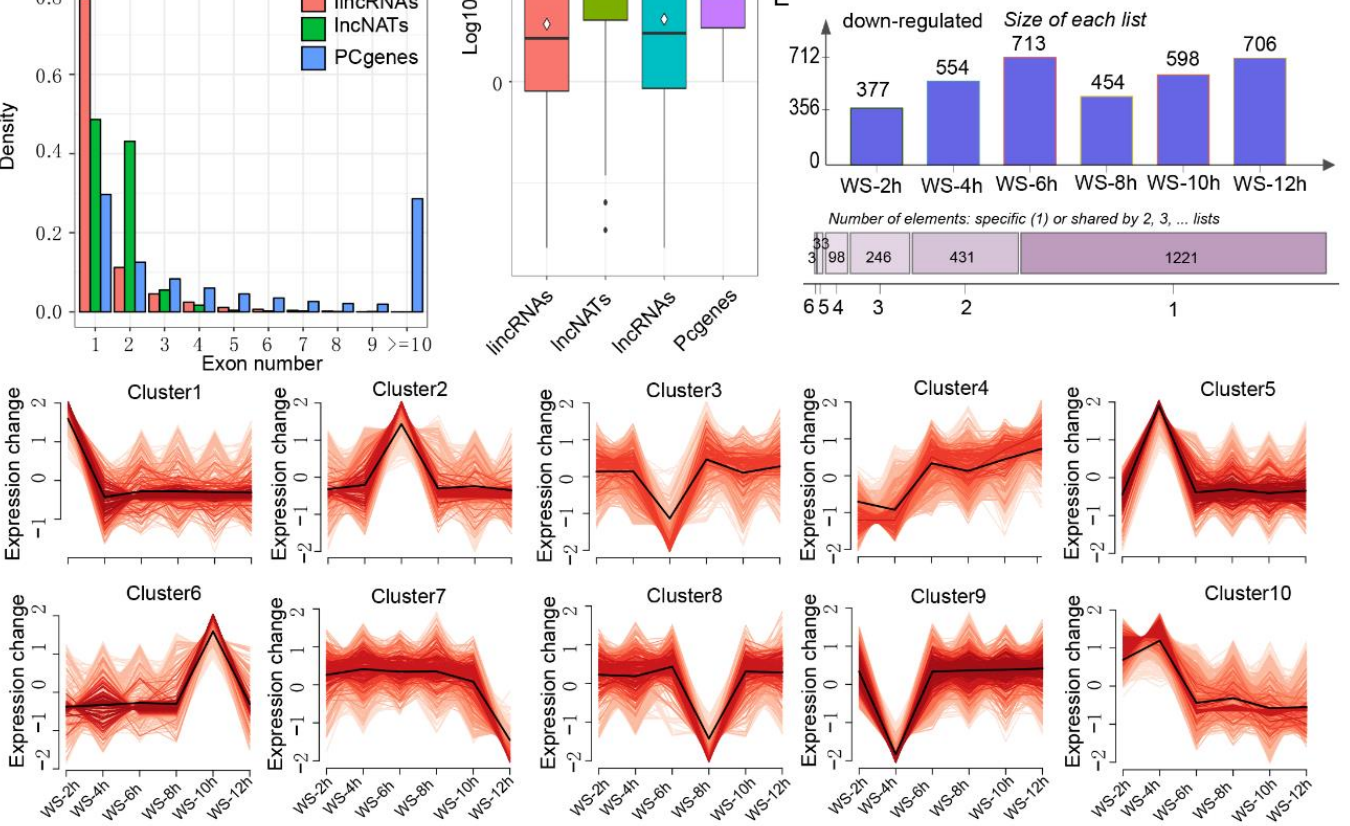

Figure 3. Characterizations of long non-coding RNA (lncRNAs) in maize root tips. (A) Length density distributions of long intergenic non-coding RNAs (lincRNAs), long non-coding natural antisense transcripts (lncNATs), and protein-coding genes (PCgenes). (B) Distributions of exon numbers in lincRNA, lncNATs and PCgenes. (C) The boxplot shows the expression level of lincRNAs, lncNATs, lncRNAs, and PCgenes. (D) The numbers of up-regulated lncRNAs after after $2 \mathrm{~h}$ (WS_2h), $4 \mathrm{~h}$ (WS_4h), $6 \mathrm{~h}$ (WS_6h), $8 \mathrm{~h}$ (WS_8h), $10 \mathrm{~h}$ (WS_10h), and $12 \mathrm{~h}$ (WS_12h) of stress. (E) The number of down-regulated lncRNAs in the samples of WS_2h, WS_4h, WS_6h, WS_8h,WS_10h, and WS_12h. (F) The expression clusters of differentially expressed lncRNAs based on the 'mfuzz' package in the R software.

To identify the differentially expressed lncRNAs (DElncRs) that responded to WS conditions, the expression of lncRNA under stress was compared with expression under normal conditions (WS_0h). 
A total of 3190 DElncRs were identified in at least one stress condition (Supplemental Data Set S2), which included over half the proportion of identified lncRNAs, indicating that the expression level of lncRNAs was significantly induced by WS conditions. The up-regulated lncRNAs ranged from 373 at Ws_2h to 757 at WS_6h, and 788 lncRNAs were identified in more than two stress conditions (Figure 3D). The down-regulated lncRNAs had similar performance to up-regulated lncRNAs, and 811 lncRNAs were detected in more than two stress conditions (Figure 3E). These DElncRs were clearly classified into ten clusters based on a normalized $\log _{2}$ (foldchange), in which the specific expression tendency of lncRNAs was presented. For example, Cluster2 was specifically up-regulated at WS_6h, and Cluster9 was specifically down-regulated at WS_4h (Figure 3F).

\subsection{Association of the Expression Between IncRNAs and DEpcGs}

To exploit the potential function of the lncRNAs involved in WS responses, a weighted gene co-expression network analysis (WGCNA) was performed. This analysis obtained 11 distinct modules shown in the dendrogram, in which major tree branches are labeled with different colors to highlight the different modules (Figure 4A). A total of 6035 lncRNAs were identified to be involved in 11 modules, ranging from one in the 'purple' module to 2677 in the 'grey' module (Table S5), indicating that the lncRNAs participated in the DEpcG regulation network. The modules closely related to WS response were of particular interest in this present study. Thus, the correlations between the modules and distinct samples were calculated, and we found that all the modules except 'pink' were significantly $(p<0.05)$ associated with at least one of the WS samples (Figure 4B). An enrichment analysis of the DEpcGs in each WS-responded module was further conducted to identify the key metabolic pathways involving lncRNAs, and DEpcGs in five modules were significantly enriched, including 'blue' (EC2.7.11.1, MAPK signaling pathway), 'brown' (EC1.4.3.21, Phenylalanine metabolism; EC4.3.1.24, Phenylpropanoid biosynthesis), 'red' (EC2.5.1.99, Carotenoid biosynthesis), and 'black' (EC4.3.1.24, Phenylpropanoid biosynthesis), all of which play vital roles in the WS response. The DEpcGs and DElncRs involved in these pathways were screened for a further analysis according to their significant associations. For example, 'blue' was significantly related to WS_4h, and then the DElncRs at WS_4h were selected (Table S6).

The 'brown' module was negatively related with WS_4h, and 22 DEpcGs and 35 DElncRs associated with the enriched pathway were down-regulated (Figure 4C). Most of these DEpcGs, including three genes (Zm00001d003015, Zm00001d051166, and Zm00001d017276) encoding phenylalanine ammonia-lyase and four genes (Zm00001d024740, Zm00001d024753, Zm00001d007161, and Zm00001d008300) encoding peroxidase. Over half of the DElncRs (such as TCONS_00043110, TCONS_00077962, TCONS_00084669, and TCONS_00105920) had high eigengene connectivity. The 'blue' group was positively associated with WS_4h; seven up-regulated DEpcGs were involved in the MAPK signaling pathway, and 64 DElncRs were up-regulated at WS_4h (Figure 4D). Of the seven DEpcGs, six genes (Zm00001d012263 encoded the serine/threonine-protein kinase SAPK4, Zm00001d016924 encoded the ETHYLENE INSENSITIVE 3-like 5 protein, Zm00001d020939 encoded the EIL transcription factor, Zm00001d037719 encoded the CAPIP1, Zm00001d041920 encoded the transcription factor PosF21, and Zm00001d047017 encoded putative HLH DNA-binding domain superfamily protein had higher connectivity to lncRNAs, whereas only a few DElncRs, such as TCONS_00166326,TCONS_00060596, and TCONS_00149876, had a higher connection. Only one DEpcG (Zm00001d018819, encoding nine-cis-epoxycarotenoid dioxygenase 5) was involved in carotenoid biosynthesis, and 28 down-regulated DElncRs at WS_4h were detected in the 'red' module (Figure 4E), which were connected with more than 15 DElncRs. The 'black' module was positively associated with WS_12h, and 12 up-regulated DEpcGs and 10 up-regulated DElncRs were identified (Figure 4F). All of these EDpcGs encoded peroxidase, and most of them had relative higher epigen gene connectivity than DElncRs, except TCONS_00177501. These results collectively demonstrated that the expression of lncRNAs was regulated in a similar way with PCgenes involved in these key pathways to respond to WS conditions. 
A

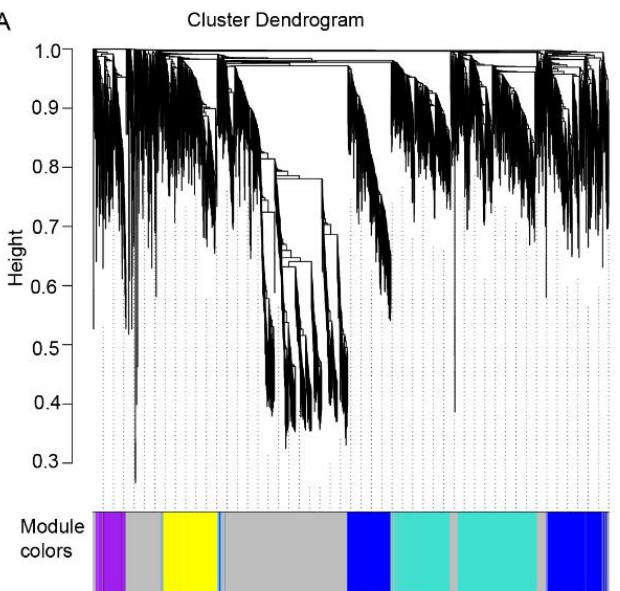

c

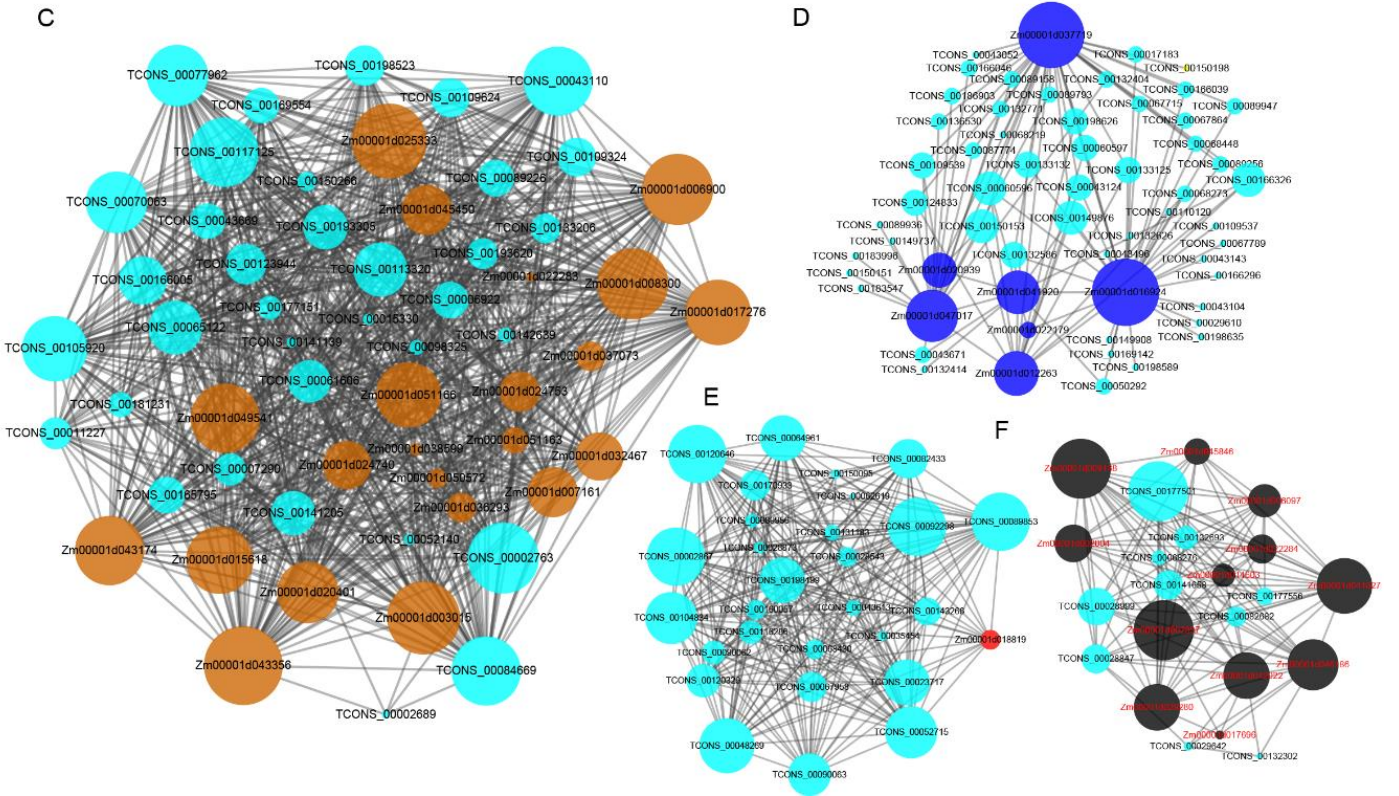

B

Module-trait corretions

\begin{tabular}{|c|c|c|c|c|c|c|c|}
\hline blue & $\begin{array}{r}-0.55 \\
0.01\end{array}$ & $\begin{array}{r}-0.29 \\
(0.2)\end{array}$ & $(8 \mathrm{e}-06)$ & 0.2 & -0.23 & 0.055 & $\begin{array}{c}0.0067 \\
0\end{array}$ \\
\hline \multirow[t]{2}{*}{ turquoise } & -0.099 & -0.074 & 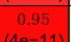 & -0.16 & -0.26 & -0.074 & -0.28 \\
\hline & $(0.7)$ & & & & & & \\
\hline \multirow[t]{2}{*}{ yellow } & $\begin{array}{l}0.4 \\
(0.07)\end{array}$ & $\begin{array}{r}0.031 \\
(0.9)\end{array}$ & $\begin{array}{c}0.75 \\
(8 e-05)\end{array}$ & $\begin{array}{l}-0.25 \\
0.33\end{array}$ & $\begin{array}{l}-0.17 \\
0.5 .5\end{array}$ & $\begin{array}{r}-0.36 \\
0.11\end{array}$ & $\begin{array}{l}-0.41 \\
0.072\end{array}$ \\
\hline & $\begin{array}{l}0.71 \\
30-04)\end{array}$ & $\begin{array}{l}0.24 \\
0.31\end{array}$ & -0.63 & -0.33 & 0.15 & -0.12 & -0.028 \\
\hline & 0.81 & 0.31 & -0.28 & -0.17 & -0.0053 & -0.32 & -0.34 \\
\hline & $(9 \mathrm{e}-06)$ & $(0.2)$ & $(0.2)$ & $(0.5)$ & (1) & $0.2)$ & $(0.1)$ \\
\hline & 0.71 & 0.46 & -0.48 & -0.02 & -0.24 & -0.2 & -0.22 \\
\hline & $(3 \mathrm{e}-04)$ & $(0.04)$ & $(0.03)$ & & $(0.3)$ & (0.4) & (0.3) \\
\hline purple & $\begin{array}{l}0.089 \\
(0.7)\end{array}$ & $\begin{array}{c}0.011 \\
\text { (1) }\end{array}$ & $\begin{array}{c}-0.9 \\
(3 e-08)\end{array}$ & $\begin{array}{r}-0.036 \\
(0.9)\end{array}$ & $\begin{array}{l}0.25 \\
0.33)\end{array}$ & $\begin{array}{l}0.23 \\
0.33\end{array}$ & $\begin{array}{l}0.35 \\
0.11\end{array}$ \\
\hline \multirow{4}{*}{ green } & -0.35 & 0.35 & -0.49 & 0.56 & -0.38 & 0.17 & 0.14 \\
\hline & $(0.1)$ & $(0.1)$ & $(0.02)$ & $(0.008)$ & $(0.09)$ & (0.5) & (0.6) \\
\hline & -0.54 & -0.38 & -0.36 & 0.078 & 0.27 & 0.42 & 0.52 \\
\hline & $(0.01)$ & $(0.09)$ & $(0.1)$ & (0.7) & $(0.2)$ & $(0.06)$ & \\
\hline \multirow{3}{*}{ magenta } & $\begin{array}{l}(0.02) \\
(0.02)\end{array}$ & $\begin{array}{c}-0.21 \\
(0.4)\end{array}$ & $\begin{array}{l}-0.41 \\
(0.07)\end{array}$ & $\begin{array}{l}0.49 \\
(0.03)\end{array}$ & $\begin{array}{l}(0.3) \\
(0.3)\end{array}$ & $\begin{array}{l}0.41 \\
(0.06)\end{array}$ & $\begin{array}{l}0.45 \\
(0.04)\end{array}$ \\
\hline & -0.14 & -0.16 & & -0.16 & -0.18 & -0.16 & -0.19 \\
\hline & & $(0.5)$ & & $(0.5)$ & (0.4) & $(0.5)$ & (0.4) \\
\hline
\end{tabular}

Figure 4. Co-expression network of the transcripts, including the protein-coding genes (PCgenes) and long non-coding RNA (lncRNAs) involved in waterlogging stress in maize root tips. (A) Hierarchical cluster tree and color bands indicating the 11 modules identified by the weighted gene co-expression network (WGCNA). (B) Analysis of the module-trait association. Each row represents a module, and each column represents a sample under waterlogging stress (WS) conditions. The numbers on the top and bottom of each cell represent the correlation and significant p-values, respectively. (C) The significantly enriched PCgenes (DEpcGs) involved in phenylpropanoid biosynthesis and the corresponding co-expressed differentially expressed lncRNAs (DElncRs) in the 'brown' module. (D) The significantly enriched DEpcGs involved in MAPK signaling and the corresponding co-expressed DElncRs in the 'blue' module. (E) The significantly enriched DEpcGs involved in carotenoid biosynthesis and the corresponding co-expressed DElncRs in the 'red' module. (F) The significantly enriched DEpcGs involved in phenylpropanoid biosynthesis and the corresponding co-expressed DElncRs in the 'black' module.

\subsection{The Expression of DElncRs Is Positively Associated with Waterlogging Tolerance}

To verify the expression levels of DEpcGs and DElncRs in each module, 20 DEpcGs and 18 DElncRs were randomly selected to quantify their expression in B73 seedling root tips using qRT-PCR. The expression levels of DEpcGs and DElncRs were in agreement with the RNA-Seq data and the qRT-PCR experiment (Figure 5A, Tables S7 and S8), and significantly higher correlations 
between them were detected (Figure 5B,C). In order to analyze the co-expression profile of DEpcGs and DElncRs in the same module among 32 different genotypes, four pairs of DEpcGs and DElncRs (Zm00001d029280 and TCONS_00177501 in 'black'; Zm00001d012263 and TCONS_00124833 in 'blue'; Zm00001d015618 and TCONS_00105920 in 'brown'; Zm00001d018819 and TCONS_00092298 in 'red') were chosen to analyze their expressions among the 32 maize inbred lines at WS_0h and WS_4h; the expression correlation among the phenotypes of survival rate (SR) after a long-term WS condition [48] for the DEpcGs and DElncRs in each pair was calculated. High correlations between DEpcGs and DElncRs, ranging from 0.58 to 0.72 , were commonly displayed in the four modules, indicating that the co-expression of PCgenes-IncRNA occurred among different genotypes in response to WS conditions (Figure 5D, Table S9). The expression of DEpcGs and DElncRs was also significantly correlated with the phenotype of SR, for which Zm00001d029280 and TCONS_00177501 in the "black" module were negatively correlated with SR, and the other three DEpcGs and three DElncRs in the other three modules were positively correlated with SR, suggesting their roles in waterlogging tolerance. These data further indicated that the expression of IncRNAs (such as TCONS_00177501) and PCgenes (such as Zm00001d029280) may coordinate the tolerance under waterlogging condition.

A

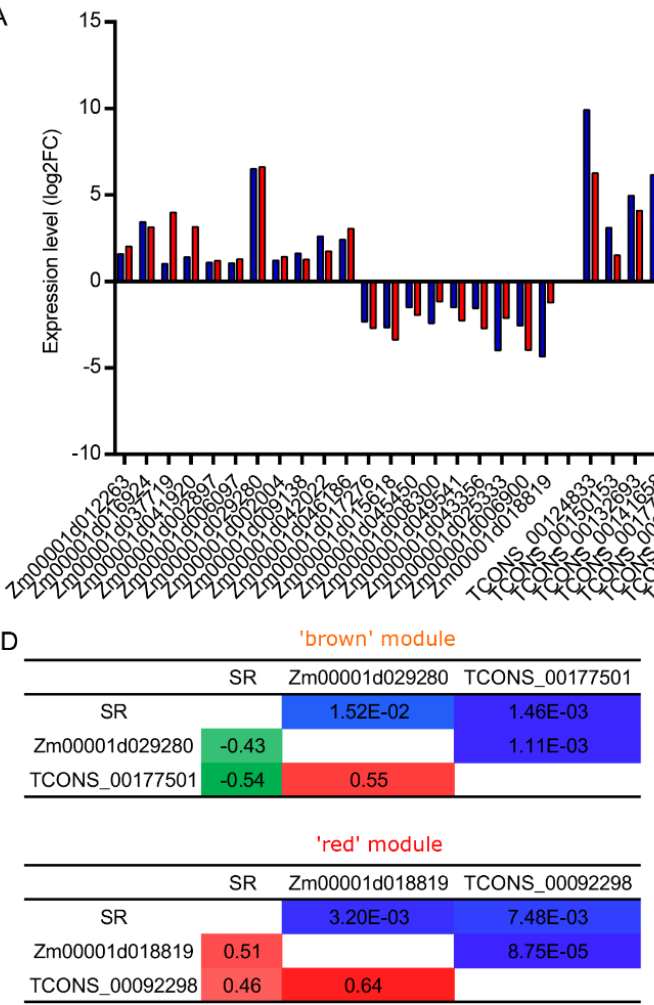

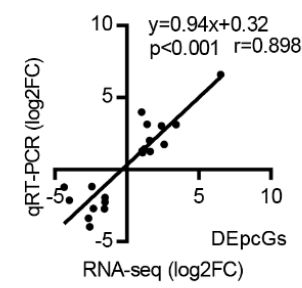

C

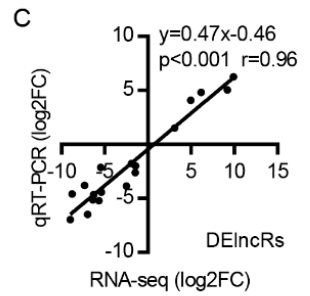

'blue' module
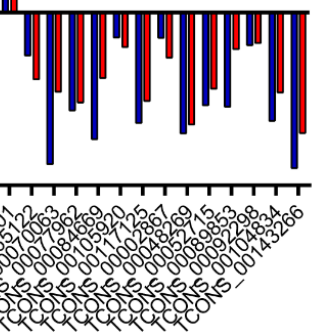

\begin{tabular}{|c|c|c|c|}
\hline & $\mathrm{SR}$ & Zm00001d012263 & TCONS_00124833 \\
\hline SR & & 3.05E-06 & 4.73E-04 \\
\hline Zm00001d012263 & 0.72 & & 3.31E-06 \\
\hline \multirow[t]{3}{*}{ TCONS_00124833 } & 0.58 & 0.72 & \\
\hline & & 'black' module & \\
\hline & SR & Zm00001d015618 & TCONS_00105920 \\
\hline SR & & 1.77E-03 & $3.62 \mathrm{E}-02$ \\
\hline Zm00001d015618 & 0.53 & & 5.81E-04 \\
\hline TCONS_00105920 & 0.49 & 0.58 & \\
\hline
\end{tabular}

Figure 5. The expression levels of protein-coding genes (PCgenes) and long non-coding genes (lncRNAs) in maize seedlings after $4 \mathrm{~h}$ waterlogging stress (WS). (A) qRT-PCR verification of the expression of 20 differentially expressed PCgenes (DEpcGs) and 18 differentially expressed lncRNAs (DElncRs) in B73 seedlings after a $4 \mathrm{~h}$ WS condition. (B) The expression correlation between the qRT-PCR and RNA-seq of the 20 DEpcGs in B73 seedlings after a $4 \mathrm{~h}$ WS condition. (C) The expression correlation between qRT-PCR and RNA-seq of the 18 DElncRs in B73 seedlings after a $4 \mathrm{~h}$ WS condition. (D) The expression correlation of DEpcG and DElncR in the 'brown', 'blue', 'red', and 'black' modules among the 32 different maize genotypes after a $4 \mathrm{~h}$ WS condition are shown. The lower triangle indicates the correlation coefficient, and the upper triangle indicates the p-value of the correlation. The relative expressions of each DEpcG and DElncR in each genotype were calculated based on the control samples (not stress), which were further transformed through the function $\log _{2}()$. FC, foldchange; SR, survival rate of 32 maize genotypes, which were taken from Yu et al. (2019) [48]. 


\subsection{Most of the DElncRs Were Localized within the Previously Mapped Quantitative Trait Loci (QTL)}

The DElncRs localized within known QTL regions could also be considered candidates to regulate waterlogging tolerance. We thus investigated the DElncRs involved in key WS-responding metabolic pathways in 'blue', 'brown', 'red', and 'black'. A total of 137 DElncRs (Table S6) were mapped to 10 chromosomes, and approximately 59\% (81 of 137) of DElncRs were localized within the previously mapped QTL, of which only the DElncRs in chromosome 9 were not within any QTL regions (Figure 6). Moreover, about 47\% (38 of 81) of co-localized DElncRs were mapped within more than two QTL regions. For example, the TCONS_00092298 in chromosome 4 is located within three QTL [63-65] associated with six phenotype traits (PH, RA2nd, SDW, TDW, and RDW), TCONS_00131183 in chromosome 5 is located within four QTL $[29,63,66,67]$ controlling the phenotypes of PH and RA, and TCONS_00089956 in chromosome 3 is located within three QTL $[63,68]$ controlling the RA, GY, and RLod. These results demonstrate that DElncRs may also affect phenotype variations in response to WS.

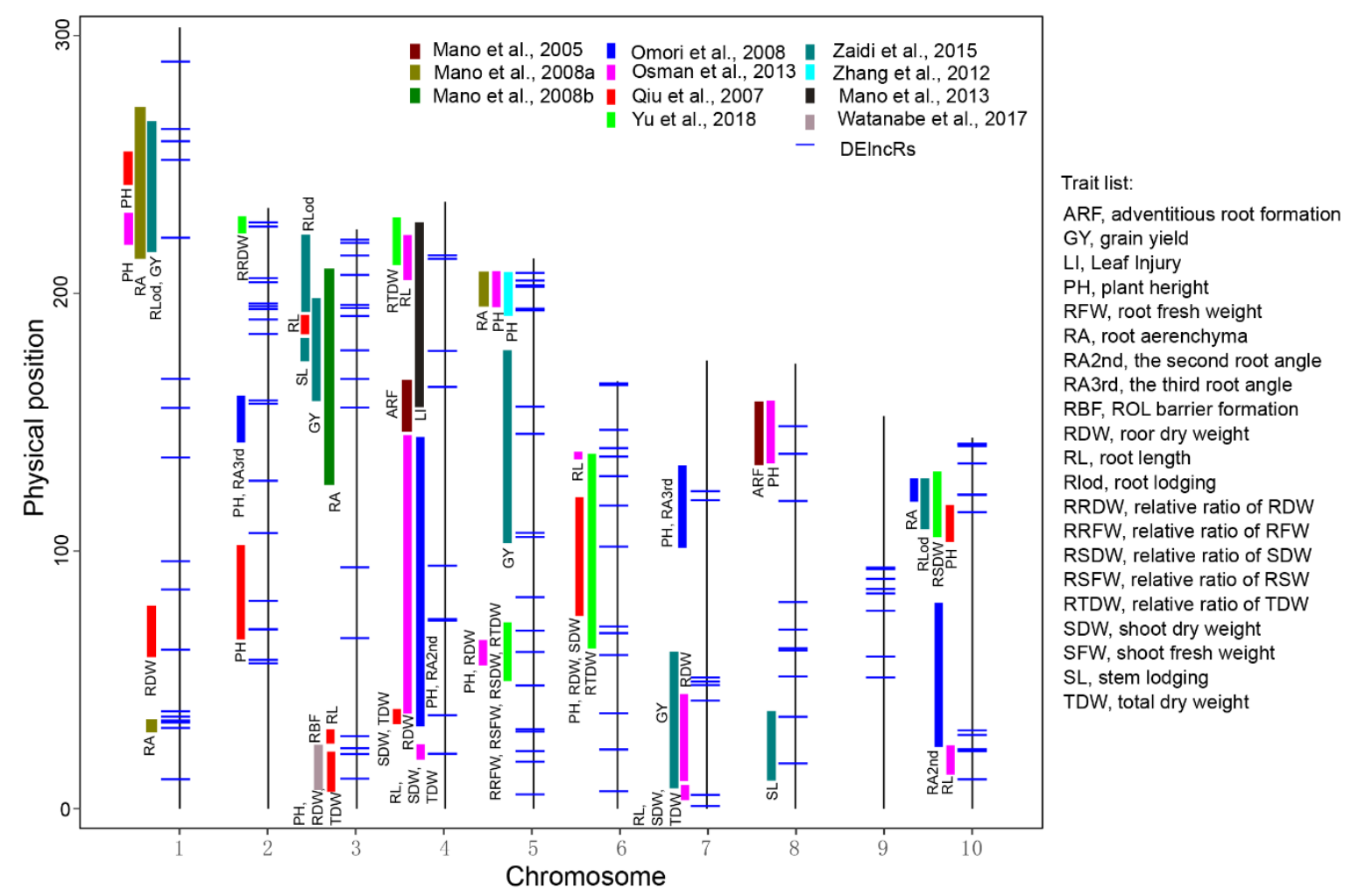

Figure 6. Co-localization of the differentially expressed long non-coding RNA (DElncRs) with previously identified quantitative trait loci (QTL) associated with waterlogging tolerance. The rectangular box represents the QTL interval, and the different colors indicate the different investigations.

\subsection{Conserved Anoxic Motif in the DElncR Promoter}

A total of 145 up-regulated DElncRs were ultimately identified to analyze their conserved motifs in the promoter region. The elements responding to three stresses were discovered; $88.3 \%$ (128 of 145), 43.4\% (63 of 128), and 31.0\% (45\%) of these DElncRs had anoxic (anoxia response element, ARE; GC-motif), drought (MBS), and low-temperature (LTR) properties, respectively (Table 1). A few DElncRs had more than two anoxic elements; for example, TCONS_00051666 had five ARE motifs, TCONS_00034141 had four GC-motifs, and TCONS_00043489 had one ARE and one GC-motif. Moreover, the responsiveness elements of abscisic acid, MeJA, auxin, gibberellin acid, and salicylic acid were also found in the DElncRs promoter, covering DElncRs ranging from $21.4 \%$ to $77.9 \%$. These data suggest that the DElncRs were responding to stress and hormone stimuli, specifically under anoxic conditions. 
Table 1. The stress- and hormone-responsive elements in the $1.5 \mathrm{~kb}$ promoter region of the high-confidence hypoxia response of long non-coding RNA.

\begin{tabular}{cccc}
\hline Responsiveness & Element & Number of DElncRs & Ratio (\%) \\
\hline Anaerobic & ARE, GC-motif & 128 & 88.3 \\
Drought & MBS & 63 & 43.4 \\
Low-temperature & LTR & 45 & 31.0 \\
Abscisic acid & ABRE & 113 & 77.9 \\
Auxin & AuxRR-core, TGA-element & 63 & 43.4 \\
Gibberellin acid & TATC-box, GARE-motif, P-box & 75 & 51.7 \\
MeJA & TGACG-motif & 99 & 68.3 \\
Salicylic acid & TCA-element & 31 & 21.4 \\
\hline
\end{tabular}

\section{Discussion}

Investigating the mechanisms of gene regulation underlying WS conditions will contribute to a better understanding of the molecular basis for waterlogging tolerance and help maize adapt better to WS. Although the transcriptomic response involved in waterlogging conditions has been studied in previous works $[10,11,45]$, there are few investigations focusing on early response, especially for the regulatory lncRNAs that participate in low-oxygen metabolism. The overall goal of the present study was to determine the transcriptional responses associated with the early stages of WS and characterize the role of the lncRNAs involved in key hypoxia-metabolic pathways via an RNA-Seq approach. Thus, the root samples of B73 seedlings subjected to WS for less than $12 \mathrm{~h}$ were collected, and the expressions of PCgenes and lncRNAs were cataloged, which revealed the important role of DElncRs in hypoxia-signaling in response to WS. The results of our comprehensive transcriptome analysis will help expand our knowledge on metabolic pathways during waterlogging in maize.

The major characteristic of waterlogged organs is their dramatically decreased concentration of oxygen, which leads to the alteration of the metabolic pathway from aerobic to anaerobic respiration $[1,3]$. The physiological responses, such as accumulated hydrogen peroxide will cause toxicity to plant cells, but the expressions of genes encoding antioxidant/metabolic enzymes are rapidly induced to maintain homeostasis. Signal transduction, protein degradation, ion transport, and carbon and amino acid metabolism played important roles during the late stage of WS (12-24 h) [11]. Furthermore, energy-production, programmed cell death, aerenchyma formation, and ethylene responsiveness were the main pathways adapted to long-term WS (5 days) [10]. The analysis of early responses to waterlogging in the present study also demonstrated that the expressions of a large number of genes changed, and DEpcGs were enriched in vital metabolic pathways (Figure 1, Table S2). These DEpcGs were classified into nine clusters, among which three clusters (Clusters 1,3, and 6) were significantly enriched in different KEGG pathways (Figure 1, Figure S1), and eight clusters were enriched in GO terms (Figures S2-S9). The glycolysis/gluconeogenesis pathway, which produces energy and recycles carbon for other pathways to survive, was found to be up-regulated, indicating this pathway's underlying central role in WS in early, late, and long-term responses. For example, six up-related genes, including Zm00001d017121 encoded glyceraldehyde-3-phosphate dehydrogenase 4, Zm00001d012103 encoded aldolase2, Zm00001d034256 encoded phosphohexose isomerase1, Zm00001d028759 encoded pyruvate decarboxylase isozyme 3, Zm00001d037689 encoded hexokinase7, and Zm00001d010588 encoded pyruvate decarboxylase1. The genes involved in glycolysis and fermentation in the present study were also up-regulated after five days WS in the tolerant line HKI1105 [10]. The phenylpropanoid biosynthesis pathway was significantly enriched, in which 22 genes were down expressed, including seven peroxidases (Zm00001d007952, Zm00001d022282, Zm00001d022283, Zm00001d024752, Zm00001d037547, Zm00001d037550, Zm00001d046035, and Zm00001d050572), which are enzyme scavengers for reactive oxygen species (ROS), demonstrating that ROS metabolism was also regulated during the early stage. Moreover, ethylene biosynthesis related genes, including six 1-aminocyclopropane-1-carboxylate (ACC) oxidase, such as Zm00001d024843, Zm00001d024850, and Zm00001d024851, which were up-regulated 
during the early stage, as well as one up-regulated ACC synthase (Zm00001d026060), were significantly enriched in the cysteine and methionine metabolism pathway. Ethylene signaling controls oxygen sensing under hypoxia conditions. A previous study showed that many genes were involved in the ethylene-response related pathway [10], in which nine genes, such as Zm00001d027302 encoding the SKP1-like protein 21, Zm00001d002896 encoding TUB-transcription factor 4, and Zm00001d032849 encoding RING/U-box superfamily protein (Table S2), adapted to long-term stress were up-regulated during the early response. These data collectively demonstrated that the hypoxia response pathways were activated during early stress conditions. Interestingly, four down-regulated genes involved in benzoxazinoid biosynthesis and 10 genes involved in the $(\alpha-)$ linolenic acid metabolism pathways were also significantly enriched, suggesting that they may play important roles in regulating early responses to WS.

Transcription factors, like DNA binding proteins, are involved in various growth, development, and stress response processes. Previous studies on Arabidopsis have reported that heat shock factors (HSFs), ethylene response-binding proteins, MADS-box proteins, the AP2 domain, leucine zipper, zinc finger, and WRKY factors increased in response to oxygen deprivation $[6,7,60,61]$. A comparative transcriptome analysis of tolerant and sensitive lines in maize and rice also demonstrated that ethylene response-binding proteins play a vital role in enhancing tolerance [9,45]. A total of 39 up-regulated AP2-EREBP genes, the major downstream components of ethylene signaling, were significantly enriched in the present study (Figure 2), among which eight genes belonged to group VII ERFs, implying the important roles of their responses to hypoxia in maize, as described in Arabidopsis and rice [62]. Moreover, a significant enrichment of the transcription factors of the bZIP, MYB, NAC, bHLH, AUX-IAA, LOB, HB, and GRAS genes was also detected (Figure 2), suggesting that multiple TF families are also directly involved in responses to low-oxygen in maize root tips.

Many studies have been conducted to investigate the transcriptomes of PCgenes in response to WS condition in plants such as Arabidopsis, rice, maize, cotton, and soybean $[7,9,11,69,70]$. However, there are few investigations centered on lncRNAs responding to WS. Indeed, lncRNAs are a diverse class of RNAs engaged in numerous biological processes [16]. With the development of high-throughput sequencing techniques, increasing numbers of functional lncRNAs have been identified, which, together with PCgenes, have been employed to reveal the high level of complexity of eukaryotic transcriptomes [71]. In this research, maize inbred line B73 was used to identify WS-related lncRNAs, and a total of 6099 high-confidence lncRNAs were identified in the roots, of which 5662 were lincRNAs and 436 were lncNATs; these quantities are far more than those reported by Li et al. [21], which may be due to the tissue specificity and updated reference genome of maize. Over half of these lncRNAs (3190) were differentially expressed under WS and the ten clear expression tendencies of DElncRs were clustered (Figure 3), indicating that lncRNAs with different catalogs directly responded to WS, which provides proof of lncRNA's involvement in low-oxygen responses.

One of the main objectives of this study was to determine whether lncRNAs are involved in the regulation of key metabolic pathways in response to WS in root tips. A co-expression network analysis of DEpcGs and lncRNAs was conducted and showed that 10 of 11 modules were significantly associated with at least one WS trait (Figure 4), suggesting the potential association between PCgenes and lncRNAs in regulating hypoxia response. Remarkably, four modules ('brown', 'blue', 'red', and 'black') were significantly enriched in three metabolic pathways, including phenylpropanoid biosynthesis, MAPK signaling, and carotenoid biosynthesis, which are popular pathways that respond to hypoxia [1]; 137 DElncRs were involved in these pathways, indicating their vital roles in regulated hypoxia-related signaling. The association of DEpcGs and DElncRs was further verified among different maize genotypes (Figure 5), and the tight association between them clarified that DElncRs are co-expressed with DEpcGs in diverse lines. The expression of DEpcGs and DElncRs was also significantly associated with the waterlogging tolerant phenotype (Figure 5). Interestingly, most of these DElncRs were co-localized with previously identified QTL (Figure 6), suggesting that these DElncRs are the possible candidates to control the corresponding traits associated with waterlogging 
tolerance. Moreover, most (88.3\%) of the 148 high-confident hypoxia response lncRNAs (highly induced by WS) had anoxic response elements, such as ARE and GT-box, in their promoters, providing the impression that hypoxia-induced lncRNAs have similar response methods to PCgenes, suggesting the possibility that they could manipulate DElncRs. These clues may imply that hypoxia-induced lncRNAs that were similar to the expression of PCgenes involved in hypoxia-related pathways also affect waterlogging tolerance in maize seedlings.

Plants have evolved the endogenous circadian clock to anticipate day and night changes, which has a profound influence on numerous biological processes such as gene expression, enzyme activity, metabolism, and stress response [72]. Accumulating evidence has demonstrated that core regulators of the circadian clock rhythm in Arabidopsis, CIRCADIAN CLOCK ASSOCIATED 1 (CCA1) and LATE-ELONGATED HYPOCOTYL (LHY) of morning-phase, are directly involved in the regulation of abiotic stresses such as cold response [73]. In our present study, Zm00001d049543 encoding CCA1 and Zm00001d024546 encoding LHY had the highest expression at WS_0h and rhythmically down-regulated until WS_12h (Table S2). These expression profiles were similar to CCA1 in Arabidopsis under free-running condition during the daytime [74], implicating that WS imposed on B73 seedlings may not disrupt the rhythm of the circadian clock. Further investigations through a side-by-side time-point control would help us distinguish the expression difference caused by WS and the rhythm of the circadian clock. Nevertheless, our analysis presented here generated a relatively robust list of PCgenes and lncRNAs that respond to WS during the early stage in maize root tips, which will likely be useful for future functional genomics research and precipitate more comprehensive studies on gene regulation under hypoxia conditions. These findings contribute new knowledge to our understanding of the hypoxia regulatory network in plants.

Supplementary Materials: The following are available online at http://www.mdpi.com/2073-4425/11/3/267/s1, Figure S1: A heatmap displaying the expression of protein-coding genes after waterlogging stress (WS) involved in the significantly enriched metabolic pathway in Cluster1, Cluster3, and Cluster6, Figure S2: The significantly enriched Gene Ontology of the biological process for protein-coding genes in Cluster1, Figure S3: The significantly enriched Gene Ontology of biological process for protein-coding genes in Cluster2, Figure S4: The significantly enriched Gene Ontology of the biological process for protein-coding genes in Cluster3. The top 30 items are shown, Figure S5: The significantly enriched Gene Ontology of the biological process for protein-coding genes in Cluster4. The top 30 items are shown, Figure S6: The significantly enriched Gene Ontology of the biological process for protein-coding genes in Cluster5, Figure S7: The significantly enriched Gene Ontology of the biological process for protein-coding genes in Cluster6. The top 30 items are shown, Figure S8: The significantly enriched Gene Ontology of biological process for protein-coding genes in Cluster7, Figure S9: The significantly enriched Gene Ontology of biological process for protein-coding genes in Cluster8. The top 30 items are shown, Table S1: Data quality of transcriptome sequencing, Table S2: The TPM values of each sample of expressed Pcgenes, Table S3: The TPM values of each sample of expressed lncRNAs, Table S4: Characterizations of the identified lncRNAs, Table S5: The Pcgenes and lncRNAs presented in 11 different modules, Table S6: The list of DEpcGs and DElncRs involved in significantly enriched pathways, Table S7: The primers of PCgenes and lncRNAs used for qRT-PCR verification, Table S8: The qRT-PCR data for 20 differentially protein-coding genes and 18 differentially expressed lncRNAs in B73 seedlings, Table S9: The qRT-PCR data for 4 differentially protein-coding genes and 4 differentially expressed lncRNAs in four different modules among 32 maize inbred lines, Supplemental Data Set S1: The list of differentially expressed protein-coding genes after a $2 \mathrm{~h}, 4 \mathrm{~h}, 6 \mathrm{~h}, 8 \mathrm{~h}, 10 \mathrm{~h}$, and $12 \mathrm{~h}$ WS condition, Supplemental Data Set S2: A list of differentially expressed long non-coding RNAs after a $2 \mathrm{~h}, 4 \mathrm{~h}, 6 \mathrm{~h}, 8 \mathrm{~h}, 10 \mathrm{~h}$, and $12 \mathrm{~h}$ WS condition.

Author Contributions: Conceptualization, F.Y. and F.Q.; methodology, F.Y.; software, Z.T.; validation, T.F., K.T., and K.L.; formal analysis, Z.T. and F.Y.; investigation, F.T., K.T., and K.L.; data curation, Z.T. and F.Y.; writing-original draft preparation, F.Y. and Z.T.; writing—review and editing, F.Q.; supervision, F.Y. and F.Q.; funding acquisition, F.Q. All authors have read and agreed to the published version of the manuscript.

Funding: This research was supported by the National Natural Science Foundation of China (Project 31,801,369 and 31571675) and the National Key Research and Development Program of China (2018YFD0100101).

Conflicts of Interest: The authors declare no conflicts of interest.

\section{References}

1. Bailey-serres, J.; Fukao, T.; Gibbs, D.J.; Holdsworth, M.J.; Lee, S.C.; Licausi, F.; Perata, P.; Van Dongen, J.T. Making sense of low oxygen sensing. Trends Plant Sci. 2012, 17, 129-138. [CrossRef] [PubMed] 
2. Hirabayashi, Y.; Mahendran, R.; Koirala, S.; Konoshima, L.; Yamazaki, D.; Watanabe, S.; Kim, H.; Kanae, S. Global flood risk under climate change. Nat. Clim. Chang. 2013, 3, 816-821. [CrossRef]

3. Voesenek, L.A.; Bailey-Serres, J. Flood adaptive traits and processes: An overview. New Phytol. 2015, 206, 57-73. [CrossRef] [PubMed]

4. Fu, J.; Cheng, Y.; Linghu, J.; Yang, X.; Kang, L.; Zhang, Z.; Zhang, J.; He, C.; Du, X.; Peng, Z.; et al. RNA sequencing reveals the complex regulatory network in the maize kernel. Nat. Commun. 2013, 4, 2832. [CrossRef] [PubMed]

5. Wang, M.; Yuan, D.; Tu, L.; Gao, W.; He, Y.; Hu, H.; Wang, P.; Liu, N.; Lindsey, K.; Zhang, X. Long noncoding RNAs and their proposed functions in fibre development of cotton (Gossypium spp.). New Phytol. 2015, 207, 1181-1197. [CrossRef]

6. Lee, S.C.; Mustroph, A.; Sasidharan, R.; Vashisht, D.; Pedersen, O.; Oosumi, T.; Voesenek, L.A.; Bailey-Serres, J. Molecular characterization of the submergence response of the Arabidopsis thaliana ecotype Columbia. New Phytol. 2011, 190, 457-471. [CrossRef]

7. Van Veen, H.; Vashisht, D.; Akman, M.; Girke, T.; Mustroph, A.; Reinen, E.; Hartman, S.; Kooiker, M.; Tienderen, P.; Schranz, M.; et al. Transcriptomes of Eight Arabidopsis thaliana Accessions Reveal Core Conserved, Genotype- and Organ-Specific Responses to Flooding Stress. Plant Physiol. 2016, 172, 668-689. [CrossRef]

8. Vashisht, D.; van Veen, H.; Akman, M.; Sasidharan, R. Variation in Arabidopsis flooding responses identifies numerous putative "tolerance genes". Plant Signal. Behav. 2016, 11, e1249083. [CrossRef]

9. Minami, A.; Yano, K.; Gamuyao, R.; Nagai, K.; Kuroha, T.; Ayano, M.; Nakamori, M.; Koike, M.; Kondo, Y.; Niimi, Y.; et al. Time-course transcriptomics analysis reveals key responses of submerged deepwater rice to flooding. Plant Physiol. 2018, 176, 3081-3102. [CrossRef]

10. Arora, K.; Panda, K.K.; Mittal, S.; Mallikarjuna, M.G.; Rao, A.R.; Dash, P.K.; Thirunavukkarasu, N. RNAseq revealed the important gene pathways controlling adaptive mechanisms under waterlogged stress in maize. Sci. Rep. 2017, 7, 10950. [CrossRef]

11. Zou, X.; Jiang, Y.; Liu, L.; Zhang, Z.; Zheng, Y. Identification of transcriptome induced in roots of maize seedlings at the late stage of waterlogging. BMC Plant Biol. 2010, 10, 189. [CrossRef] [PubMed]

12. Kreuzwieser, J.; Hauberg, J.; Howell, K.A.; Carroll, A.; Rennenberg, H.; Millar, A.H.; Whelan, J. Differential response of gray poplar leaves and roots underpins stress adaptation during hypoxia. Plant Physiol. 2009, 149, 461-473. [CrossRef] [PubMed]

13. Christianson, J.A.; Llewellyn, D.J.; Dennis, E.S.; Wilson, I.W. Global gene expression responses to waterlogging in roots and leaves of cotton (Gossypiumhirsutum L.). Plant Cell Physiol. 2010, 51, 21-37. [CrossRef]

14. Mustroph, A.; Lee, S.C.; Oosumi, T.; Zanetti, M.E.; Yang, H.; Ma, K.; Yaghoubi-Masihi, A.; Fukao, T.; Bailey-Serres, J. Cross-kingdom comparison of transcriptomic adjustments to low-oxygen stress highlights conserved and plant- specific responses. Plant Physiol. 2010, 152, 1484-1500. [CrossRef]

15. Narsai, R.; Rocha, M.; Geigenberger, P.; Whelan, J.; van Dongen, J.T. Comparative analysis between plant species of transcriptional and metabolic responses to hypoxia. New Phytol. 2011, 190, 472-487. [CrossRef]

16. Quinn, J.J.; Chang, H.Y. Unique features of long non-coding RNA biogenesis and function. Nat. Rev. Genet. 2016, 17, 47-62. [CrossRef]

17. Liu, J.; Jung, C.; Xu, J.; Wang, H.; Deng, S.; Bernad, L.; Arenas-Huertero, C.; Chua, N.H. Genome-wide analysis uncovers regulation of long intergenic noncoding RNAs in Arabidopsis. Plant Cell 2012, 24, 4333-4345. [CrossRef]

18. Wang, H.; Chung, P.J.; Liu, J.; Jang, I.C.; Kean, M.J.; Xu, J.; Chua, N.H. Genome-wide identification of long noncoding natural antisense transcripts and their responses to light in Arabidopsis. Genome Res. 2014, 24, 444-453. [CrossRef]

19. Liu, H.; Wang, R.; Mao, B.; Zhao, B.; Wang, J. Identification of lncRNAs involved in rice ovule development and female gametophyte abortion by genome-wide screening and functional analysis. BMC Genom. 2019, 20, 90. [CrossRef]

20. Zhang, Y.C.; Liao, J.Y.; Li, Z.Y.; Yu, Y.; Zhang, J.P.; Li, Q.F.; Qu, L.H.; Shu, W.S.; Chen, Y.Q. Genome-wide screening and functional analysis identify a large number of long noncoding RNAs involved in the sexual reproduction of rice. Genome Biol. 2014, 15, 512. [CrossRef] [PubMed] 
21. Li, L.; Eichten, S.R.; Shimizu, R.; Petsch, K.; Yeh, C.T.; Wu, W.; Chettoor, A.M.; Givan, S.A.; Cole, R.A.; Fowler, J.E.; et al. Genome-wide discovery and characterization of maize long non-coding RNAs. Genome Biol. 2014, 15, R40. [CrossRef]

22. Xu, W.; Yang, T.; Wang, B.; Han, B.; Zhou, H.; Wang, Y.; Li, D.Z.; Liu, A. Differential expression networks and inheritance patterns of long non-coding RNAs in castorbean seeds. Plant J. 2018, 95, 324-340. [CrossRef] [PubMed]

23. Deng, P.C.; Liu, S.; Nie, X.J.; Weining, S.; Wu, L. Conservation analysis of long non-coding RNAs in plants. Sci. China Life Sci. 2018, 61, 190-198. [CrossRef] [PubMed]

24. Ulitsky, I. Evolution to the rescue: Using comparative genomics to understand long non-coding RNAs. Nat. Rev. Genet. 2016, 17, 601-614. [CrossRef] [PubMed]

25. Swiezewski, S.; Liu, F.; Magusin, A.; Dean, C. Cold-induced silencing by long antisense transcripts of an Arabidopsis Polycomb target. Nature 2009, 462, 799-802. [CrossRef]

26. Heo, J.B.; Sung, S. Vernalization-mediated epigenetic silencing by a long intronic noncoding RNA. Science 2010, 331, 76-79. [CrossRef]

27. Franco-Zorrilla, J.M.; Valli, A.; Todesco, M.; Mateos, I.; Puga, M.I.; Rubio-Somoza, I.; Leyva, A.; Weigel, D.; García, J.A.; Paz-Ares, J. Target mimicry provides a new mechanism for regulation of microRNA activity. Nat. Genet. 2007, 39, 1033-1037. [CrossRef]

28. Rymarquis, L.A.; Kastenmayer, J.P.; Huttenhofer, A.G.; Green, P. Diamonds in the rough: mRNA-like non-coding RNAs. Trends Plant Sci. 2008, 13, 329-334. [CrossRef]

29. Campalans, A.; Kondorosi, A.; Crespi, M. Enod40, a short open reading framecontaining mRNA, induces cytoplasmic localization of a nuclear RNA binding protein in Medicago truncatula. Plant Cell 2004, 16, 1047-1059. [CrossRef]

30. Gultyaev, A.P.; Roussis, A. Identification of conserved secondary structures and expansion segments in enod40 RNAs reveals new enod40 homologues in plants. Nucleic Acids Res. 2007, 35, 3144-3152. [CrossRef]

31. Ding, J.; Lu, Q.; Ouyang, Y.; Mao, H.; Zhang, P.; Yao, J.; Xu, C.; Li, X.; Xiao, J.; Zhang, Q. A long noncoding RNA regulates photoperiodsensitive male sterility, an essential component of hybrid rice. Proc. Natl. Acad. Sci. USA 2012, 109, 2654-2659. [CrossRef] [PubMed]

32. Wang, Y.; Luo, X.J.; Sun, F.; Hu, J.H.; Zha, X.J.; Su, W.; Yang, J.S. Overexpressing lncRNA LAIR increases grain yield and regulates neighbouring gene cluster expression in rice. Nat. Commun. 2018, 9, 3516. [CrossRef] [PubMed]

33. Fang, J.; Zhang, F.; Wang, H.; Wang, W.; Zhao, F.; Li, Z.; Sun, C.; Chen, F.; Xu, F.; Chang, S.; et al. Ef-cdlocus shortens rice maturity duration without yield penalty. Proc. Natl. Acad. Sci. USA 2019, 116, 18717-18722. [CrossRef] [PubMed]

34. Wang, J.; Meng, X.; Dobrovolskaya, O.B.; Orlov, Y.L.; Chen, M. Non-coding RNA and their roles in stress response in plants. Genom. Proteom. Bioinf. 2017, 15, 301-312. [CrossRef] [PubMed]

35. Yu, Y.; Zhou, Y.F.; Feng, Y.Z.; He, H.; Lian, J.P.; Yang, Y.W.; Lei, M.Q.; Zhang, Y.C.; Chen, Y.Q. Transcriptional landscape of pathogen-responsive lncRNA in rice unveils the role of ALEX1 in jasmonate pathway and disease resistance. Plant Biotechnol. J. 2019. [CrossRef]

36. Qin, T.; Zhao, H.; Cui, P.; Albesher, N.; Xiong, L. A nucleus-localized long non-coding RNA enhances drought and salt stress tolerance. Plant Physiol. 2017, 175, 1321-1336. [CrossRef]

37. Chen, L.; Shi, S.; Jiang, N.; Khanzada, H.; Wassan, G.M.; Zhu, C.; Peng, X.; Xu, J.; Chen, Y.; Yu, Q.; et al. Genome-wide analysis of long non-coding RNAs affecting roots development at early stage in the rice response to cadmium stress. BMC Genom. 2018, 19, 460. [CrossRef]

38. Chen, M.; Wang, C.; Bao, H.; Chen, H.; Wang, Y. Genome-wide identification and characterization of novel lncRNA in Populus under nitrogen deficiency. Mol. Genet. Genom. 2015, 291, 1663-1680. [CrossRef]

39. Gong, L.C.; Xu, H.M.; Guo, G.L.; Zhang, T.; Shi, J.W.; Chang, C. Long Non-coding RNA H19 protects H9c2 cells against hypoxia-induced injury by targeting MicroRNA-139. Cell Physiol. Biochem. 2017, 44, 857-869. [CrossRef]

40. Li, L.; Wang, Q.; Yuan, Z.; Chen, A.; Liu, Z.; Li, H.; Wang, Z. Long non-coding RNA H19 contributes to hypoxia-induced CPC injury by suppressing Sirt1 through miR-200a-3p. Acta Biochim. Biophys. Sin. 2018, 50, 950-959. [CrossRef] 
41. Bhan, A.; Deb, P.; Shihabeddin, N.; Ansari, K.I.; Brotto, M.; Mandal, S.S. Histone methylase MLL1 coordinates with HIF and regulate lncRNA HOTAIR expression under hypoxia. Gene 2017, 629, 16-28. [CrossRef] [PubMed]

42. Wu, Z.; Zhao, S.; Li, C.; Liu, C. LncRNA TUG1 serves an important role in hypoxia-induced myocardial cell injury by regulating the miR-145-5p-Binp3 axis. Mol. Med. Rep. 2018, 17, 2422-2430. [CrossRef] [PubMed]

43. Li, X.; Deng, S.J.; Zhu, S.; Jin, Y.; Cui, S.P.; Chen, J.Y.; Xiang, C.; Li, Q.Y.; He, C.; Zhao, S.F.; et al. Hypoxia-induced lncRNA-NUTF2P3-001 contributes to tumorigenesis of pancreatic cancer by derepressing the miR-3923/KRAS pathway. Oncotarget 2016, 7, 6000-6014. [CrossRef]

44. Wu, J.; Okada, T.; Fukushima, T.; Tsudzuki, T.; Sugiura, M.; Yukawa, Y. A novel hypoxic stress-responsive long non-coding RNA transcribed by RNA polymerase III in Arabidopsis. RNA Biol. 2012, 9, 302-313. [CrossRef] [PubMed]

45. Thirunavukkarasu, N.; Hossain, F.; Mohan, S.; Shiriga, K.; Mittal, S.; Sharma, R.; Singh, R.K.; Gupta, H.S. Genome-wide expression of transcriptomes and their co-expression pattern in subtropical maize (Zea mays L.) under waterlogging stress. PLoS ONE 2013, 8, e70433. [CrossRef] [PubMed]

46. Yu, F.; Liang, K.; Fang, T.; Zhao, H.; Han, X.; Cai, M.; Qiu, F. A group VII ethylene response factor gene, ZmEREB180, coordinates waterlogging tolerance in maize seedlings. Plant Biotechnol. J. 2019, 17, 2286-2298. [CrossRef]

47. Bolger, A.M.; Lohse, M.; Usadel, B. Trimmomatic: A flexible trimmer for Illumina sequence data. Bioinformatics 2014, 30, 2114-2120. [CrossRef]

48. Kim, D.; Langmead, B.; Salzberg, S.L. HISAT: A fast spliced aligner with low memory requirements. Nat. Methods 2015, 12, 357. [CrossRef]

49. Liao, Y.; Smyth, G.K.; Shi, W. featureCounts: An efficient general purpose program for assigning sequence reads to genomic features. Bioinformatics 2013, 30, 923-930. [CrossRef]

50. Langmead, B.; Salzberg, S.L. Fast gapped-read alignment with Bowtie 2. Nat. Methods 2012, 9, 357. [CrossRef]

51. Trapnell, C.; Williams, B.A.; Pertea, G.; Mortazavi, A.; Kwan, G.; van Baren, M.J.; Salzberg, S.L.; Wold, B.J.; Pachter, L. Transcript assembly and quantification by RNA-Seq reveals unannotated transcripts and isoform switching during cell differentiation. Nat. Biotechnol. 2010, 28, 511. [CrossRef] [PubMed]

52. Li, B.; Dewey, C.N. RSEM: Accurate transcript quantification from RNA-Seq data with or without a reference genome. BMC Bioinform. 2011, 12, 323. [CrossRef] [PubMed]

53. Love, M.I.; Huber, W.; Anders, S. Moderated estimation of fold change and dispersion for RNA-seq data with DESeq2. Genome Boil. 2014, 15, 550. [CrossRef] [PubMed]

54. Benjamini, Y.; Hochberg, Y. Controlling the false discovery rate: A practical and powerful approach to multiple testing. J. R. Stat. Soc. Ser. B (Methodol.) 1995, 57, 289-300. [CrossRef]

55. Kumar, L.; Futschik, M.E. Mfuzz: A software package for soft clustering of microarray data. Bioinformation 2007, 2, 5. [CrossRef] [PubMed]

56. Du, Z.; Zhou, X.; Ling, X.; Zhang, Z.; Su, Z. agriGO: A GO analysis toolkit for the agricultural community. Nucleic Acids Res. 2010, 38, W64-W70. [CrossRef]

57. Langfelder, P.; Horvath, S. WGCNA: An R package for weighted correlation network analysis. BMC Bioinform. 2008, 9, 559. [CrossRef]

58. Shannon, P.; Markiel, A.; Ozier, O.; Baliga, N.S.; Wang, J.T.; Ramage, D.; Amin, N.; Schwikowski, B.; Ideker, T. Cytoscape: A software environment for integrated models of biomolecular interaction networks. Genome Res. 2003, 13, 2498-2504. [CrossRef]

59. Livak, K.J.; Schmittgen, T.D. Analysis of relative gene expression data using real-time quantitative PCR and the 2(-Delta Delta C(T)) method. Methods 2001, 25, 402-408. [CrossRef]

60. Lescot, M.; Déhais, P.; Thijs, G.; Marchal, K.; Moreau, Y.; De Peer, Y.V.; Rouzé, P.; Rombauts, S. PlantCARE, a database of plant cis-acting regulatory elements and a portal to tools for in silico analysis of promoter sequences. Nucleic Acids Res. 2002, 30, 325-327. [CrossRef]

61. Jiao, Y.; Peluso, P.; Shi, J.; Liang, T.; Stitzer, M.C.; Wang, B.; Campbell, M.S.; Stein, J.C.; Wei, X.; Chin, C.S.; et al. Improved maize reference genome with single-molecule technologies. Nature 2017, 546, 524-527. [CrossRef] [PubMed]

62. Gibbs, D.J.; Conde, J.V.; Berckhan, S.; Prasad, G.; Mendiondo, G.M.; Holdsworth, M.J. Group VII ethylene response factors coordinate oxygen and nitric oxide signal transduction and stress responses in plants. Plant Physiol. 2015, 169, 23-31. [CrossRef] [PubMed] 
63. Mano, Y.; Omori, F. Verification of QTL controlling root aerenchyma formation in a maize $\times$ teosinte" Zea nicaraguensis" advanced backcross population. Breed. Sci. 2008, 58, 217-223. [CrossRef]

64. Mano, Y.; Omori, F.; Kindiger, B.; Takahashi, H. A linkage map of maize $\times$ teosinte Zea luxurians and identification of QTLs controlling root aerenchyma formation. Mol. Breed. 2008, 21, 327-337. [CrossRef]

65. Omori, F.; Mano, Y. QTL mapping of root angle in $\mathrm{F}_{2}$ populations from maize 'B73' $\times$ teosinte 'Zea luxurians'. Plant Root 2008, 1, 57-65. [CrossRef]

66. Qiu, F.; Zheng, Y.; Zhang, Z.; Xu, S. Mapping of QTL associated with waterlogging tolerance during the seedling stage in maize. Ann. Bot. 2007, 99, 1067-1081. [CrossRef]

67. Zhang, X.; Tang, B.; Yu, F.; Li, L.; Wang, M.; Xue, Y.; Zhang, Z.; Yan, J.; Yue, B.; Zheng, Y.; et al. Identification of major QTL for waterlogging tolerance using genome-wide association and linkage mapping of maize seedlings. Plant Mol. Biol. Report. 2012, 31, 594-606. [CrossRef]

68. Zaidi, P.H.; Rashid, Z.; Vinayan, M.T.; Almeida, G.D.; Phagna, R.K.; Babu, R. QTL mapping of agronomic waterlogging tolerance using recombinant inbred lines derived from tropical maize (Zea mays L.) germplasm. PLoS ONE 2015, 10, e0124350. [CrossRef]

69. Zhang, Y.; Kong, X.; Dai, J.; Luo, Z.; Li, Z.; Lu, H.; Xu, S.; Tang, W.; Zhang, D.; Li, W.; et al. Global gene expression in cotton (Gossypium hirsutum L.) leaves to waterlogging stress. PLoS ONE 2017, 12, e0185075. [CrossRef]

70. Lin, Y.; Li, W.; Zhang, Y.; Xia, C.; Liu, Y.; Wang, C.; Xu, R.; Zhang, L. Identification of genes/proteins related to submergence tolerance by transcriptome and proteome analyses in soybean. Sci. Rep. 2019, 9, 14688. [CrossRef] [PubMed]

71. Kapusta, A.; Feschotte, C. Volatile evolution of long noncoding RNA repertoires: Mechanisms and biological implications. Trends Genet. 2014, 30, 439-452. [CrossRef] [PubMed]

72. Lu, H.; Mcclung, C.R.; Zhang, C. Tick Tock: Circadian Regulation of Plant Innate Immunity. Annu. Rev. Phytopathol. 2017, 55, 287-311. [CrossRef] [PubMed]

73. Greenham, K.; Mcclung, C.R. Integrating circadian dynamics with physiological processes in plants. Nat. Rev. Genet. 2015, 16, 598-610. [CrossRef] [PubMed]

74. Wu, J.; Tsai, H.; Joanito, I.; Wu, Y.; Chang, C.; Li, Y.; Wang, Y.; Hong, J.; Chu, J.; Hsu, C.; et al. LWD-TCP complex activates the morning gene CCA1 in Arabidopsis. Nat. Commun. 2016, 7, 13181. [CrossRef] 